\title{
Making Diamond Qubits Talk to Light
}

\section{A solid-state qubit satisfies three key requirements of a building block for a quantum network.}

\section{by Kai-Mei Fu*}

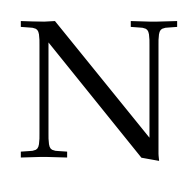
etworks of quantum-entangled qubits (Fig. 1) could transform communication and computation, enabling a fundamentally secure quantum internet [1] or a universal quantum computer [2]. Photons seem to be the best option for connecting the qubits, or "nodes," because they can travel great distances along well-defined paths through waveguides, fibers, or free space. For the node, there are many more contenders, including trapped ions, trapped atoms, warm atomic vapors, quantum dots, rare-earth dopants in a crystal, and defects in diamond called nitrogen vacancy centers. However, none of these options are ideal, and each must satisfy multiple physical requirements at once. Three of the most important criteria have now been met by a relative newcomer to the qubit arena-a silicon point defect embedded in diamond. Focusing on one such defect, Christian Nguyen and colleagues at Harvard University report that they can read out its state in a single shot, make it communicate efficiently

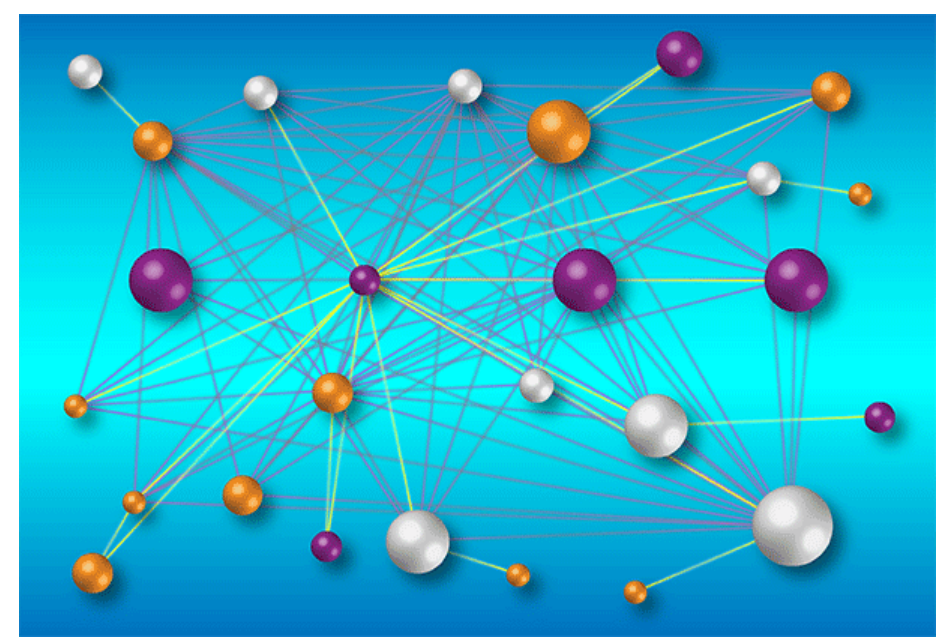

Figure 1: Quantum networks could enable a more secure internet or quantum computers. (APS/Alan Stonebraker) ${ }^{*}$ Department of Electrical and Computer Engineering, University of
Washington, Seattle, WA, USA with photons, and couple it to a long-lived quantum memory $[2,3]$. This demonstration is the first time all three of these conditions have been met together in a solid-state system, which is considered the most scalable qubit option.

Efficient communication with photons is a prerequisite for any candidate node. Various schemes for this communication exist. But the most practical ones are deterministic, meaning the arriving photon's quantum state will always be transferred to the node, and the node will always transfer its state to an outgoing photon. Such schemes require a condition known as high cooperativity, C. (Cooperativity is the ratio between the square of photon-qubit coupling and the product of the photon and qubit decoherence rates.) Trapped-ion and trapped-atom platforms have led the progress with deterministic qubit-photon interfaces [5-7]. But making large numbers of qubits may be easiest with solid-state systems, so there is a drive to get them into the high-cooperativity regime.

Researchers have achieved high cooperativity in solidstate qubits. But an unrealized goal is to combine this feature with long-time information storage (quantum memory) in a multiqubit system, or "register." In a register, different qubits read/write and store information, a division of labor that's essential for achieving large-scale networks. Quantum memory times approaching 1 minute have been demonstrated with nitrogen vacancy (NV) centers in a tenqubit register [8] (see 11 September 2019 Synopsis.) But integrating NV centers into a nanophotonic device to enable a strong interaction with light was found to degrade the defects' optical properties, limiting their use to nondeterministic photon-qubit interactions [9]. On the other hand, high cooperativities have been obtained in quantumdot systems [10] but not in combination with a long quantum memory.

The experiments from Nguyen and colleagues indicate that this powerful combination is possible with a different type of diamond defect, known as the negatively charged silicon vacancy $\left(\mathrm{SiV}^{-}\right)$center $[3,4]$. Here, a silicon atom replaces two carbon atoms in the diamond lattice, sitting symmetrically between the vacancy sites. The silicon vacancy has an effective electron spin-1/2, which serves as a quantum bit. The researchers introduced these $\mathrm{SiV}^{-}$defects into a thin diamond bar-micrometers long and hundreds of nanometers wide-that was perforated with regularly spaced holes (Fig. 2). This hole pattern created an optical 


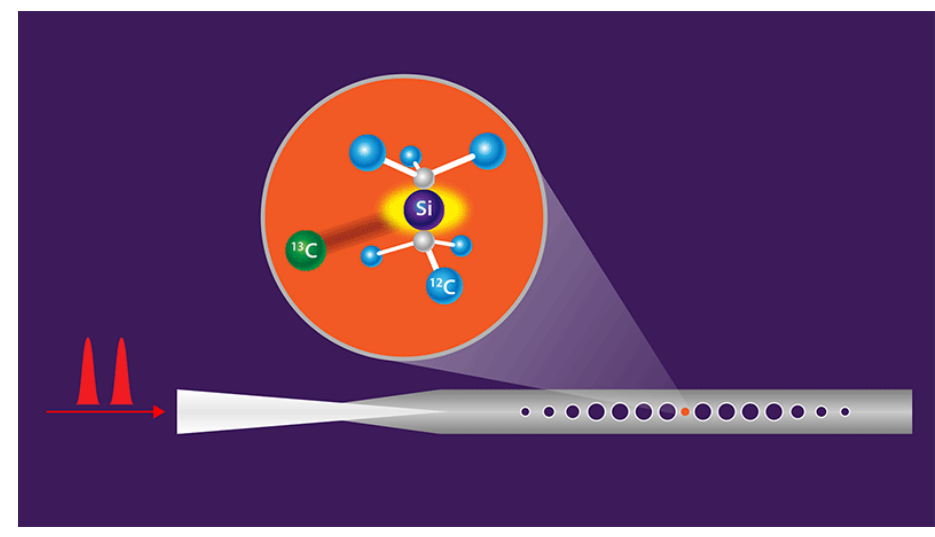

Figure 2: The diamond nanocavity (grey bar with holes) designed by Nguyen and colleagues [3, 4]. The cavity fosters a strong coupling between light from a fiber and the electron of an $\mathrm{SiV}^{-}$defect embedded in the diamond (inset). This coupling allows the photon state (encoded in the photon's arrival time) to be reliably transferred to the electron spin. (C. T. Nguyen et al. [4]; adapted by APS/Alan Stonebraker)

cavity for a particular mode of light that was sent in via an optical fiber to interact with the $\mathrm{SiV}^{-}$.

Compared with the NV center, $\mathrm{SiV}^{-}$has inversion symmetry and is therefore significantly more resilient to electricfield noise present in nanophotonic devices. Thanks to this resilience, the Harvard team achieved a high cooperativity $(C>30)$, enabling the electronic state to be read out in a single measurement with high fidelity. The high cooperativity also allowed them to transfer a photonic quantum state-here encoded in the arrival time of the photon-to the $\mathrm{SiV}^{-}$electronic state. However, this type of encoding precluded a deterministic photon-qubit interaction.

Transfer of the photon's state to the qubit requires that the electron spin remain coherent during the transfer and while the state is stored. To meet this condition, the researchers had to keep their qubit at very low temperatures (below $500 \mathrm{mK}$ ), necessitating specialized cooling techniques. During the photon-state transfer experiment, they stored the transferred state on the electron for $20 \mu \mathrm{s}$, which is a short memory time. But in separate experiments, the team showed they could extend this memory to $1 \mathrm{~ms}$ by applying sequences of microwave pulses that effectively cancel external magnetic noise. They extended the memory further still, to $200 \mathrm{~ms}$, by transferring the electronic $\mathrm{SiV}^{-}$state to a nearby carbon-13 nuclear spin, thus forming a two-qubit register. This step is usually accomplished by combining the hyperfine interaction between the spins with radio-frequency drives on each spin, but the driving process heats the $\mathrm{SiV}^{-}$ qubit and destroys its coherence. Nguyen and co-workers avoided the heating problem by designing a state-transfer approach that doesn't require the radio-frequency drives. The downside of this approach is a relatively low fidelity.
Quantum technologies are only practical if they are scalable. It is thus necessary to discover, engineer, and control quantum systems that can perform several tasks very well. The Harvard group demonstrates that multiple tasks are possible with the $\mathrm{SiV}^{-}$center, elevating the qubit to the level of other serious contenders for a quantum network. But there is a caveat: their demonstration of photon-electron transfer and of electron-nuclear transfer are performed on the same device, but not during the same experiments. Achieving photon-nuclear transfer in consecutive steps, as desired, will require higher fidelities. The next challenge will be entangling many $\mathrm{SiV}^{-}$centers via photons and controlling them well enough to enable a multiqubit network. For the systems we know, such as $\mathrm{NV}^{-}$and $\mathrm{SiV}^{-}$centers, this challenge is formidable, and for $\mathrm{SiV}^{-}$, there is the added complication that it is only viable at low temperatures. That's why the community must continue to ask whether there is another defect with more favorable properties or if there are better ways to control the qubits we have now.

This research is published in Physical Review Letters and Physical Review B.

\section{REFERENCES}

[1] H. J. Kimble, "The quantum internet," Nature 453, 1023 (2008).

[2] R. Raussendorf and H. J. Briegel, "A one-way quantum computer," Phys. Rev. Lett. 86, 5188 (2001).

[3] C. T. Nguyen et al., "Quantum network nodes based on diamond qubits with an efficient nanophotonic interface," Phys. Rev. Lett. 123, 183602 (2019).

[4] C. T. Nguyen et al., "An integrated nanophotonic quantum register based on silicon-vacancy spins in diamond," Phys. Rev. B 100, 165428 (2019).

[5] S.-Kai Liao et al., "Satellite-to-ground quantum key distribution," Nature 549, 43 (2017).

[6] A. Stute, B. Casabone, B. Brandstätter, K. Friebe, T. E. Northup, and R. Blatt, "Quantum-state transfer from an ion to a photon," Nat. Photon. 7, 219 (2013).

[7] A. Reiserer and G. Rempe, "Cavity-based quantum networks with single atoms and optical photons," Rev. Mod. Phys. 87, 1379 (2015).

[8] C. E. Bradley, J. Randall, M. H. Abobeih, R. C. Berrevoets, M. J. Degen, M. A. Bakker, M. Markham, D. J. Twitchen, and T. H. Taminiau, "A ten-qubit solid-state spin register with quantum memory up to one minute," Phys. Rev. X 9, 031045 (2019).

[9] P. C. Humphreys, N. Kalb, J. P. J. Morits, R. N. Schouten, R. F. L. Vermeulen, D. J. Twitchen, M. Markham, and R. Hanson, "Deterministic delivery of remote entanglement on a quantum network," Nature 558, 268 (2018).

[10] K. Hennessy, A. Badolato, M. Winger, D. Gerace, M. Atatüre, S. Gulde, S. Fält, E. L. Hu, and A. Imamoğlu, "Quantum nature of a strongly coupled single quantum dot-cavity system," Nature 445, 896 (2007).

10.1103/Physics.12.117 